\title{
Sperm Selection Using Three Semen Processing Techniques
}

\author{
Vivian Berkenbroch Ramos ${ }^{1}$, Daniela da Cunha Cipriani ${ }^{1}$, Eduardo Schuchowsky Araújo ${ }^{1}$, Rafael Alonso \\ Salvador $^{1}$, Alfred Paul Senn², Marcel Frajblat ${ }^{3}$, Vera Lucia Lângaro Amaral ${ }^{1}$
}

${ }^{1}$ University of Vale do Itajai (UNIVALI), Itajai, Santa Catarina, Brazil

${ }^{2}$ Fondation F.A.B.E.R., Lausanne, Switzerland

${ }^{3}$ Federal University of Rio de Janeiro (UFRJ), Brazil

This study was presented as a poster at the Brazilian Congress of Assisted Reproduction (SBRA, 2014)

\begin{abstract}
Objective: This study aimed to assess the efficiency, in terms of recovered motile spermatozoa with normal morphology, of three sperm selection techniques: migration-sedimentation (SS), swim-up from fresh semen (SF), and swim-up from washed (SL) sperm.

Methods: Samples from 20 normozoospermic men were divided into three equal aliquots and processed in parallel. SS was performed in a Jondet tube, using $1 \mathrm{ml}$ of semen and $2.5 \mathrm{ml}$ of Human Tubal Fluid medium (HTF+10\% Synthetic Serum Supplement, Irvine, USA). For SF, $1 \mathrm{ml}$ of HTF was layered over $1 \mathrm{ml}$ of fresh semen (SF). For SL, $1 \mathrm{ml}$ of sperm was first centrifuged ( $300 \mathrm{~g}, 10 \mathrm{~min}$ ) and the pellet resuspended in $1 \mathrm{ml}$ of HTF; a second layer of HTF was placed on top. Migration time was $1 \mathrm{~h}$ (SF and $\mathrm{SL}$ ) and $1 \mathrm{~h} 30^{\prime}$ for SS at $37^{\circ} \mathrm{C}$. After migration, $200 \mu \mathrm{l}$ were removed from the top layer (SF, SL) and from the central cone (SS). Concentration, morphology and motility were determined.

Results: Recovery rates were $25 \%$ for SS, $10.1 \%$ for SF and $4.5 \%$ for SL. SS recovery rate was significantly higher $(P<0.01)$ than the two swim-up techniques. Total motility was statistically different $(P<0.001)$, with $93.6 \%$ for SS, $91.2 \%$ for $S F$, and $77 \%$ for SL. Sperm morphology was similar between the three techniques $(P=0.12)$.

Conclusions: SS is an efficient technique for the recovery of motile spermatozoa from native semen preparations and yielded better results than SF and SL. Routine use for assisted reproduction remains to be evaluated.
\end{abstract}

Keywords: Assisted reproduction, Sperm preparation, Sperm selection, Migration-sedimentation

\section{INTRODUCTION}

With the advancement of human assisted reproduction technologies, there is a need for reliable, simple, secure methods for the selection of fertile spermatozoa (Mortimer, 2000). The increasing demand for sperm preparations in the context of intrauterine insemination (IUI) or in vitro fertilization (IVF) has provided better knowledge on how sperm contributes to the success of such techniques.

Theoretically, the ideal sperm processing technique should allow the selection of many motile spermatozoa of good quality, maintain physiological conditions, allow the removal of dead cells, microorganisms, and reactive oxygen species (ROS), avoid irreversible injuries to the sperm membrane, enable the processing of large semen volumes, and be quick, affordable and safe (Henkel \& Schill, 2003). The most used modern sperm selection methods include swim-up and discontinuous density gradient filtration (Henkel, 2012). The swim-up technique consists of carefully layering a culture medium over fresh or washed semen. With time, the motile spermatozoa that have progressively colonized the upper medium layer can be recovered by gentle aspiration. In the density gradient technique, motile spermatozoa aided by the centrifugation force pass through several layers of increasing density and are recovered in the densest bottom layer, while dead cells, debris, and microorganisms are withheld in the upper layers. The recovered motile fraction needs then to be washed twice with culture medium to remove the gradient compounds. Washing procedures are considered critical for the maintenance of sperm fertilizing ability. For instance, pelleting native semen by centrifugation may enhance ROS production from leucocytes, leading to peroxidative damage of spermatozoa and to their obliteration or reduction of their fertilizing capacity (Mortimer, 2000). In addition to the two techniques described above, the migration-sedimentation method, although described in 1985 (Tea et al., 1984), has never been widely applied. It is based on the use of a device in which motile spermatozoa that have undergone a swim-up are progressively collected in a central cone as spermatozoa are submitted to spontaneous gravitational sedimentation (Henkel \& Schill, 2003).

The purpose of our study was to compare the efficiency of recovering motile spermatozoa with normal morphology from three sperm selection techniques: migration-sedimentation (SS), swim-up from fresh semen (SF), and swim-up from washed (SL) sperm.

\section{MATERIALS AND METHODS}

Biological material and sperm analysis

Sperm samples were obtained from 20 normozoospermic men on abstinence for 2-5 days seen at an IVF clinic in Itajai, Brazil. After liquefaction, sperm concentration, motility, and morphology were determined following the WHO laboratory guidelines (WHO, 2010). Concentrations were briefly measured after a dilution of $1: 10$ with a fixative solution ( $1 \%$ formaldehyde) using a Neubauer chamber (WHO, 2010). Motility was graded in three categories: A: linear or largely circular motion, regardless of speed; $\mathrm{B}$ : local displacement without forward progression; and C: motionless. A Makler chamber was used to count a minimum of 100 spermatozoa. For morphology, smears were prepared and air-dried, and then submitted to a Diff-Quick staining procedure (Kruger et al., 1987). A single observer examined the prepared slides. Head, midpiece, and tail morphology was described as being normal or having malformations. A total of 100 spermatozoa were counted.

\section{Set-up of the migration systems}

Each sample was divided into three equal aliquots, and each was processed in parallel using three different sperm selection procedures. In all cases, HEPES-buffered Human Tubal Fluid medium (HTF, Irvine Scientific, USA) supplemented with $10 \%$ Synthetic Serum Supplement (SSS, Irvine Scientific, USA) was used. For the swim-up from fresh semen (SF) procedure, the medium was first placed on a $15-\mathrm{ml}$ conical sterile Falcon tube; semen was then gently placed underneath it using a 
A

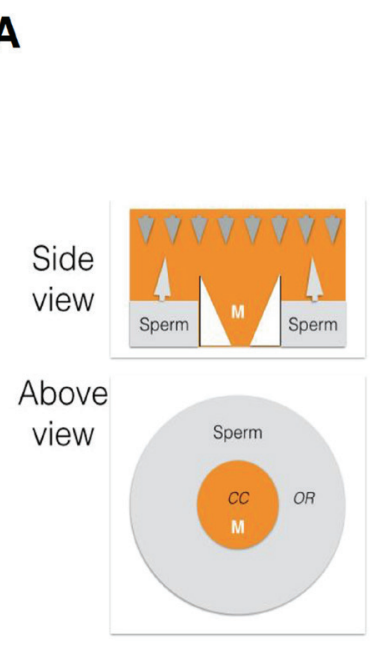

B

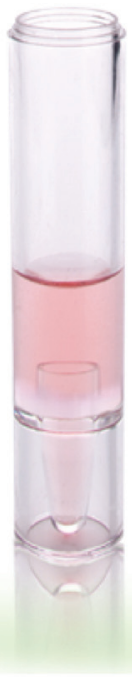

Figure 1. Schematic view of migration-sedimentation (A) and a picture of the RI-MSC ${ }^{\text {TM }}$ tube (B). Upward arrows indicate regions of migration out of the seminal plasma into the medium (M). Downward arrows indicate direction of sedimentation. OR: outer rim, CC: central cone.

Pasteur pipette at a ratio of $1: 1$. For the swim-up from washed (SL) procedure, the sperm aliquot was mixed (2:1) with washing medium and centrifuged ( 8 min, 350 g). The resulting pellet was then resuspended in HTF medium up to a volume equal to that of the sperm aliquot. A migration set-up similar to that of SF was then prepared for SL. Both the SF and SL tubes were then placed in an incubator at $37^{\circ} \mathrm{C}$ at a $45^{\circ}$ angle for one hour.

A Jondet tube (Research Instruments, England) was kindly provided by Vitrolife (São Paulo, Brazil) for the migration-sedimentation (SS) procedure. The semen aliquot was placed in the external rim of the tube, and subsequently $2.5 \mathrm{ml}$ of the HTF medium were slowly poured into the inner cone (Figure 1 ). The tube was then incubated for 1.5 hours at $37^{\circ} \mathrm{C}$.

After the migration period, $200 \mu \mathrm{l}$ were removed from the top layer (SF, SL) and from the central cone (SS), and then submitted to sperm analysis. Concentrations and motility grades were determined for each sperm suspension immediately after the end of the selection procedure.

Calculation of recovery ratios $(R)$

$R=C_{f} / C_{i} \times 100$ where $c_{i}$ and $C_{f}$ are the initial and final concentrations, respectively.

\section{Statistics}

Statistical analysis and graphic representations were produced using the Prism 6 software package (GraphPad, La Jolla, CA 92037 USA). Incidences and frequency distributions were compared using ANOVA with Tukey's test.

\section{Review board}

The research project was submitted to the Ethics Review Board of UNIVALI University in Itajaí (Comissão de Ética em Pesquisa, CEP No 644.949). Informed consent was obtained from the sperm donors as part of routine sperm analysis procedures.

\section{RESULTS}

The mean recovery and motility rates of twenty tests are presented on Table 1 . Significantly $(P<0.01)$ higher recovery rates were obtained with the SS method $(25.0 \%)$ versus swim-up techniques SF (10.1\%) and SL (5.4\%); SF and SL were not statistically different.

The percentage of motile spermatozoa $(A+B)$ was significantly lower in SL (77\%) when compared to SS (93.6\%) and SF (91.2\%). Progressive forms (A) were significantly more present in SF $(80.4 \%)$ and SS $(85.6 \%)$ when compared to SL $(57.6 \%)$, whereas non progressive forms (B) were more present in SL $(19.1 \%)$ than in SF $(10.7 \%)$ or SS $(8.0 \%)$.

The percentage of normal forms was significantly $(P=0.03)$ lower in SL $(3.5 \%)$ than in fresh semen $(5.8 \%)$, SF $(5.2 \%)$, or SS $(5.5 \%)$, and in the differentiated sperm morphology there were no significant alterations between the three tested methods (Table 2).

\section{DISCUSSION}

The results of the study showed that SF and SS were equally effective at selecting motile spermatozoa, while SS allowed the recovery of a larger amount of spermatozoa. None of the methods allowed the enrichment of normal forms in the case of normozoospermic samples. SL clearly reduced motility rates, probably as a consequence of exposure to ROS.

Future studies could look into whether the migration-sedimentation technique might be applied successfully to IUI or IVF-ICSI procedures. There are only a few studies in the literature using migration-sedimentation devices, but the ones comparing swim-up and migration-sedimentation have reported similar conclusions, in that the recovery rates of spermatozoa were higher with the migra-

Table 1. Sperm recovery rates (\%) and motility grades A and B (\%) determined after selection by three semen processing techniques: migration-sedimentation (SS), swim-up from fresh sample (SF), and swim-up from washed sample (SL), expressed as mean \pm SEM.

\begin{tabular}{|c|c|c|c|c|}
\hline Parameters & Fresh semen & SF & SL & ss \\
\hline Concentrations $\left(10^{6} / \mathrm{ml}\right)$ & $77.0 \pm 4.3$ & $10.0 \pm 15.2$ & $5.0 \pm 6.7 a$ & $22.5 \pm 33.7^{a}$ \\
\hline Recovery rates $(\%)$ & $10.1 \pm 2.4$ & $5.4 \pm 0.9^{a}$ & $25.0 \pm 5.7^{a}$ & \\
\hline \multicolumn{5}{|l|}{ Motility grades } \\
\hline$A+B(\%)$ & $77.0 \pm 11.0$ & $91.2 \pm 2.0^{a}$ & $77.0 \pm 2.8^{a, b}$ & $93.6 \pm 1.4^{b}$ \\
\hline Progressive, A (\%) & $80.5 \pm 2.4^{a}$ & $57.6 \pm 4.4^{\mathrm{a}, \mathrm{b}}$ & $85.6 \pm 2.5^{b}$ & \\
\hline Non-progressive, B (\%) & $10.7 \pm 1.4^{a}$ & $19.1 \pm 3.2^{a, b}$ & $8.0 \pm 1.5^{b}$ & \\
\hline
\end{tabular}

a,b: statistically different $(P<0.01)$

Numbers without or with different superscripts are not statistically different.

Data were compared using ANOVA and Tukey's test. 
Table 2. Sperm morphology determined after selection by three semen processing techniques: migration-sedimentation (SS), swim-up from fresh sample (SF), and swim-up from washed sample (SL), expressed as mean \pm SEM.

\begin{tabular}{|l|c|c|c|c|}
\hline \multicolumn{1}{|c|}{ Parameters } & Fresh semen & SF & SL & SS \\
\hline Normal (\%) & $5.8 \pm 0.5$ & $5.2 \pm 0.5$ & $3.5 \pm 0.4 *$ & $5.5 \pm 0.8$ \\
\hline Piriform & 9.0 & 10.4 & 11.9 \\
\hline Elongated & 14.9 & 1.2 & 0.8 & 13.6 \\
\hline Macrocephalic & 3.3 & 2.6 & 4.2 & 1.6 \\
\hline Microcephalic & 2.0 & 7.9 & 9.4 & 2.5 \\
\hline Amorphous & 7.5 & 26.6 & 26.6 & 27.9 \\
\hline Vacuoles & 27.4 & 29.5 & 28.0 & 27.4 \\
\hline Midpiece defects & 26.7 & 7.0 & 10.3 & \\
\hline Tail defects & 8.6 & & \\
\hline *. statistically different $(P<0.05)$. & & &
\end{tabular}

tion-sedimentation technique than with regular swim-up. Recovery rates ranged from 35\% (Cardona Maya et al., 2007 ) to $58 \%$ (Tea et al., 1984), but this variation may have been affected by the way recovery rates were calculated, incubation times, and whether motility was included in the formula (Cardona Maya et al., 2007 ). In the present study, recovery rates were calculated by taking into account only the number of spermatozoa. The $25.0 \%$ recovery rate for SS was significantly higher than that of both swim-up procedures $(10.1 \%$ and $5.4 \%$ for SF and $\mathrm{SL}$, respectively) (Table 1). Migration time is clearly an important factor that plays a greater role in migration-sedimentation than in swim-up, as in the latter case a plateau is reached as soon as an equilibrium between actively upwards moving and downwards sedimenting forms is reached. The amount of medium sampled after migration is another key value that might explain differences between studies; this value was set to $200 \mu \mathrm{l}$ for standardization purposes between the three tested techniques in our study.

In the present study, motility rates were equal between SF and SS (Table 2). This was also reported by other authors (Yener et al., 1990), but one study found significantly higher levels of motility using a migration-sedimentation device. Obviously, it should be pointed out that native semen quality affects recovery and motility rates of washed samples (Chan et al., 1991, Hinting et al., 2001); therefore, native semen parameters should be taken into consideration in the selection of the appropriate sperm selection procedure (Cardona Maya et al., 2007, Chan et al., 1991). In the present study, sperm motility in the SL technique was significantly lower than in SF and SS (Table 1). This illustrates the negative effects of centrifugation (Mortimer, 2000), which have been associated with the negative effects of ROS (Henkel et al., 2005). The quantification of sperm motility in this study was based on subjective parameters. Variations of $30 \%$ to $60 \%$ in the assessment of sperm motility may occur due to limitations of the human eye in quantifying the various sperm subpopulations in a sample (Verstegen et al., 2002). Computer assisted sperm analyzers (CASA) have been shown to identify motility patterns reliably (Matos et al., 2008). Although it is unclear which kinetic characteristics determined by the CASA system may be used to predict fertility or fertilization rates (Ferreira et al., 1997), the use of such a system might shed some light on the particular kinetic behaviors seen in the three analyzed methods.

Enrichment of normal forms using the swim-up procedure has led to contradictory results, with some studies showing higher values than others (Angelopoulos et al., 1998; Hammadeh et al., 2000, 2001). This might be partly due to the subjectivity of morphologi- cal evaluation, which might vary considerably between laboratories. In this study, a single observer made all determinations using Kruger's strict criteria (Kruger et al., 1986) in order to mitigate uncontrolled subjective appreciation of sperm morphology. However, here again, the quality of the native semen needs to be taken into account, as poor samples may behave differently from normozoospermic ones. Selection of motile spermatozoa is an essential step in assisted reproduction procedures, as spermatozoa need to be freed from decapacitation factors present in the seminal fluid for successful fertilization to take place. In vivo, this occurs spontaneously as the spermatozoon traverses the cervical mucus. In vitro, centrifugation and replacement of the seminal plasma by culture medium is the simplest way to achieve this goal, but it has been recognized that pelleting spermatozoa may cause the release of reactive oxygen species (ROS), which by their turn might induce loss of motility (Henkel et al., 2005), reduction of fertilization capacity, and DNA damage (Henkel et al., 2004). Swim-up from native sperm and use of density gradient have thus become the usual practice over the past decades (Mortimer, 2000). A less common technique, despite its promising features, was developed in the early 1980 s by a French group (Tea et al., 1984). It is based on the migration of motile spermatozoa into the medium followed by the recovery of spontaneously sedimenting spermatozoa into a separate receptacle. Various systems have been developed differing in shape and materials (Tea et al., 1984, Ebner et al., 2011), but all maintain a surface of contact between semen and culture medium and a receptacle where spontaneously sedimenting spermatozoa may accumulate. The most recent system reduces the sperm/medium contact to a capillary bridge created by a separate u-ring (Ebner et al., 2011). The system used in the present study is similar to a culture plate, with a central collecting well and an outer rim (Figure 1), and is quite similar to the description of a Jondet (Tea et al., 1984).

Spermatozoa may be exposed to ROS and suffer DNA damage in vivo during sperm migration into the urogenital tract (Pasqualotto et al., 2008), but also in vitro during sperm preparation procedures (Henkel, 2012). Sperm DNA fragmentation has been shown to negatively affect pregnancy rates in IUI treatments, but not in ICSI procedures (Thomson et al., 2011). However, it is generally recognized that increased sperm fragmentation is associated with increased incidence of miscarriage (Pasqualotto et al., 2002, Dar et al., 2013). Techniques that avoid centrifugation and close contact between spermatozoa and ROS producing leucocytes, such as the migration-sedimentation technique, were shown to have the potential to reduce DNA alterations (Ebner et al., 2011). 
One might conclude that the migration-sedimentation technique (SS) offers advantages over the traditional methods tested (SF or SL) in terms of sperm recovery and quality. Future studies should confirm that the migration-sedimentation technique can efficiently protect spermatozoa from molecular and structural damage, thus allowing better results in IUI or IVF-ICSI procedures.

\section{Acknowledgments}

Vitrolife (São Paulo, Brazil) kindly provided samples of migration-sedimentation tubes (RI-MSCTM from Research Instruments, England).

\section{CONFLICT OF INTERESTS}

No conflict of interest have been declared.

\section{Corresponding author:}

Vera Lucia Lângaro Amaral

Laboratory of Reproductive Biology

University of Vale do Itajai (UNIVALI)

Itajai, Santa Catarina, Brazil

E-mail:veralucia@univali.br

\section{REFERENCES}

Angelopoulos T, Moshel YA, Lu L, Macanas E, Grifo JA, Krey LC. Simultaneous assessment of sperm chromatin condensation and morphology before and after separation procedures: effect on the clinical outcome after in vitro fertilization. Fertil Steril. 1998; 69:740-7.

Cardona Maya WD, Berdugo Gutierrez JA, de los Rios J, Cadavid Jaramillo AP. Functional evaluation of sperm in CoIombian fertile men. Arch Esp Urol. 2007; 60:827-31.

Chan SY, Chan YM, Tucker MJ. Comparison of characteristics of human spermatozoa selected by the multiple-tube swim-up and simple discontinuous Percoll gradient centrifugation. Andrologia 1991; 23:213-8.

Dar S, Grover SA, Moskovtsev SI, Swanson S, Baratz A, Librach CL. In vitro fertilization-intracytoplasmic sperm injection outcome in patients with a markedly high DNA fragmentation index $(>50 \%)$. Fertil Steril. 2013; 100: 75-80.

Ebner T, Shebl O, Moser M, Mayer RB, Arzt W, Tews G. Easy sperm processing technique allowing exclusive accumulation and later usage of DNA-strandbreak-free spermatozoa. Reprod Biomed Online. 2011; 22:37-43.

Ferreira, JCP, Neves Neto JR, Papa FO. Avaliação computadorizada das características espermáticas de garanhões com fertilidade comprovada. Rev Bras Reprod Anim. 1997; 21:131-2.

Hammadeh ME, Nkemayim DC, Georg T, Rosenbaum P, Schmidt W. Sperm morphology and chromatin condensation before and after semen processing. Arch Androl. $2000 ; 44: 221-6$

Hammadeh ME, Kuhnen A, Amer AS, Rosenbaum $P$, Schmidt W. Comparison of sperm preparation methods: effect on chromatin and morphology recovery rates and their consequences on the clinical outcome after in vitro fertilization embryo transfer. Int J Androl. 2001; 24: $360-8$

Henkel RR, Schill WB. Sperm preparation for ART. Reprod Biol Endocrinol. 2003; 1:108.
Henkel R. Sperm preparation: state-of-the-art--physiological aspects and application of advanced sperm preparation methods. Asian J Androl. 2012; 14:260-9.

Henkel R, Kierspel E, Stalf T, Mehnert C, Menkveld R, Tinneberg HR, Schill WB, Kruger TF. Effect of reactive oxygen species produced by spermatozoa and leukocytes on sperm functions in non-leukocytospermic patients. Fertil Steril. 2005; 83:635-42.

Henkel R, Hajimohammad M, Stalf T, Hoogendijk C, Mehnert $C$, Menkveld R, Gips H, Schill WB, Kruger TF. Influence of deoxyribonucleic acid damage on fertilization and pregnancy. Fertil Steril. 2004; 81:965-72.

Hinting A, Lunardhi $\mathrm{H}$. Better sperm selection for intracytoplasmic sperm injection with the side migration technique. Andrologia. 2001; 33:343-6.

Kruger TF, Ackerman SB, Simmons KF, Swanson RJ, Brugo SS, Acosta AA. A quick, reliable staining technique for human sperm morphology. Arch Androl. 1987; 18:275-7.

Kruger TF, Menkveld R, Stander FS, Lombard CJ, Van der Merwe JP, van Zyl JA, Smith K. Sperm morphologic features as a prognostic factor in in vitro fertilization. Fertil Steril. 1986; 46:1118-23.

Matos DI, Araújo AA, Roberto IG, Toniolli R. Análise computadorizada de espermatozóides: revisão de literatura. Rev Bras Reprod Anim 2008; 32:225-32.

Mortimer D. Sperm preparation methods. J Androl. 2000; 21:357-66.

Pasqualotto FF, Sundaram A, Sharma RK, Borges E, Jr., Pasqualotto EB, Agarwal A. Semen quality and oxidative stress scores in fertile and infertile patients with varicocele. Fertil Steril. 2008; 89:602-7.

Pasqualotto FF, Rossi-Ferragut LM, Rocha CC, Iaconelli $A$,

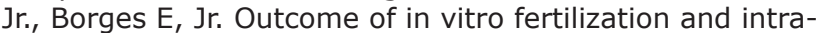
cytoplasmic injection of epididymal and testicular sperm obtained from patients with obstructive and nonobstructive azoospermia. J Urol. 2002; 167:1753-6.

Tea NT, Jondet M, Scholler R. A "migration-gravity sedimentation" method for collecting motile spermatozoa from human semen. In: Harisson RF, Bonnar J, Thompson W, eds. In Vitro Fertilization, Embryo Transfer and Early Pregnancy. Springer Netherlands; 1984. p. 117-20.

Thomson LK, Zieschang JA, Clark AM. Oxidative deoxyribonucleic acid damage in sperm has a negative impact on clinical pregnancy rate in intrauterine insemination but not intracytoplasmic sperm injection cycles. Fertil Steril. 2011; 96:843-7.

Verstegen J, Iguer-Ouada M, Onclin K. Computer assisted semen analyzers in andrology research and veterinary practice. Theriogenology. 2002; 57:149-79.

WHO Laboratory manual for the examination and processing of human semen, 5th edn. Geneva: WHO Press; 2010.

Yener C, Mathur S, Parent B. Comparison of two sperm preparation techniques using automated sperm motion analysis: migration sedimentation versus swim-up. Arch Androl. 1990; 25:17-20. 